\title{
Asymptotic expansions through the loop-tree duality
}

\author{
Judith Plenter ${ }^{\mathrm{a}} \mathbb{1}$, Germán Rodrigo ${ }^{\mathrm{b}}$ (i) \\ Instituto de Física Corpuscular, Universitat de València, Consejo Superior de Investigaciones Científicas, Parc Científic, 46980 Paterna, Valencia, \\ Spain
}

Received: 3 February 2021 / Accepted: 27 March 2021 / Published online: 15 April 2021

(C) The Author(s) 2021

\begin{abstract}
Asymptotic expansions of Feynman amplitudes in the loop-tree duality formalism are implemented at integrand-level in the Euclidean space of the loop threemomentum, where the hierarchies among internal and external scales are well-defined. The ultraviolet behaviour of the individual contributions to the asymptotic expansion emerges only in the first terms of the expansion and is renormalized locally in four space-time dimensions. These two properties represent an advantage over the method of Expansion by Regions. We explore different approaches in different kinematical limits, and derive explicit asymptotic expressions for several benchmark configurations.
\end{abstract}

\section{Introduction}

Since the Higgs boson has been discovered successfully at CERN's Large Hadron Collider almost one decade ago, clear evidence of further particles not described by the Standard Model (SM) has not been reported, although observations such as the anomalous magnetic moment of the muon [1] or the $B$ anomalies [2] hint at discrepancies between SM predictions and measurements. With decreasing experimental uncertainties it is clear that theoretical predictions at increasing precision are at the forefront of current research in order to confirm or reject deviations from the SM [3]. Consequently, higher order contributions in perturbative Quantum Field Theory (pQFT) are crucial. This endeavour quickly reaches its limits in the common approach of Dimensional Regularization (DREG) $[4,5]$. Within this technique the divergent expressions that appear in loop calculations of Feynman diagrams are regularized by working in $d=4-2 \varepsilon$ spacetime dimensions, thus leaving the problematic integrals formally well-defined. The limit $\varepsilon \rightarrow 0$ is taken only after both infrared (IR) and ultraviolet (UV) singularities have been

\footnotetext{
a e-mail: plenter@ific.uv.es (corresponding author)

b e-mail: german.rodrigo@csic.es
}

canceled in IR safe observables and/or renormalized through appropriate redefinition of the constants appearing in the theory. Within DREG, the difficulty posed by the integral(s) in a Feynman amplitude scales with the number of loops, external legs and mass scales.

The interest in asymptotic expansions within $\mathrm{pQFT}$ arises from their potential to facilitate analytic results in specific kinematic configurations, particularly when full analytic calculations in DREG are not possible. While work on the solution for further master integrals is ongoing, an expanded result can still be of great interest since it showcases the relevant behaviour of the amplitude in the needed kinematic limit. There are many observables where an analytic result is not necessary for every set of kinematics and where specific limits are the window to test potential discrepancies between experiments and SM predictions, thus identifying new physics contributions. Furthermore, in the context of the local cancellation of IR singularities expanded integrands could be very convenient to reduce computation time. While maintaining the correct analytic structure in the divergent limit and thus allowing for the combination with the realemission contributions, the less complicated form of the expanded virtual contributions is expected to evaluate faster during the point-by-point process of numerical integration.

As an example for a new-physics scenario likely to benefit from asymptotic expansions highly-boosted Higgs boson production may be mentioned: while the regime of small transverse momentum has been calculated with a point-like interaction encoding the top-quark loop [6,7], first attempts at the full calculation necessary for obtaining the large transverse momentum distribution have been published recently and rely on either numerical integration [8] or expansions in the Integration by Parts identities [9]. It is exactly this part of the amplitude which is needed in order to rule out an additional point-like effective Higgs-gluon-gluon coupling.

The interest in asymptotic expansions becomes also clear noting that there are already well-developed methods for simplifying the integrands of Feynman amplitudes. Widely 
known among them is Expansion by Regions [10-16]. While this technique has been shown to provide correct results a general proof is still pending [17]. Additionally, the degree of UV divergence rises with every term in the expansion which can be considered inconvenient.

In recent years an alternative regularization method based on the loop-tree duality (LTD) has been developed and applied both at one loop and beyond [18-36]. Other alternative methods to DREG have been proposed and are summarized in Ref. [37]. The basis of LTD is using the Cauchy residue theorem to integrate one component of the loop momentum. Loop amplitudes can thus be expressed as a sum of residues which can be reformulated as so-called dual amplitudes. These consist of sums of tree-level like objects to be integrated in what essentially is a phase-space integral.

As a result of LTD, one obtains a function to be integrated over the Euclidean three-momenta. In addition, the LTD representation exhibits a clear and localized singular structure that enables the local cancellation of IR singularities. This feature allowed the development of the Four-dimensional Unsubtraction method (FDU) [38-40]. Further, it leads to an additional characteristic: in comparison to the original Feynman amplitude as a function of Minkowski four-momenta the size of scalar products appearing in the dual integrand can be directly compared to external scales. This allows the development of a well-defined formalism of asymptotic expansions of the integrand. Specific asymptotic expansions in the context of LTD have been presented for the first time for the process $H \rightarrow \gamma \gamma$ at one loop [24]. The aim of this paper is to extend these preliminary studies to other kinematical configurations. First steps have been reported on in [41] recently.

The structure of the paper is as follows. General guidelines for the expansion of dual propagators are layed out in Sect. 2. Those rules are then applied to the scalar oneloop two-point function in Sect. 3 as well as the scalar threepoint function in Sect. 4, in both cases for a variety of limits. We aim towards obtaining an expansion that is well-defined also at integrand-level and simplifies integrands sufficiently to obtain loop analytic results at higher orders and multiple scales. For this purpose, we analyze in Sect. 5 the multiloop case of the so called Maximal Loop Topology (MLT) defined in Ref. [34], which is the most symmetric multiloop configuration and is used as the building block to construct more complex topologies.

\section{Loop-tree duality and asymptotic expansions of dual propagators}

A general one-loop scattering amplitude with $N$ external legs in the Feynman representation is given by
$\mathcal{A}_{N}^{(1)}=\int_{\ell} \mathcal{N}\left(\ell,\left\{p_{k}\right\}_{N}\right)\left(\prod_{i=1}^{N} G_{F}\left(q_{i}\right)\right)$

where the loop integral measure in $d=4-2 \varepsilon$ space-time dimensions is $\int_{\ell}=-l \mu^{4-d} \int \mathrm{d}^{d} \ell /(2 \pi)^{d}$ and the expression $\mathcal{N}\left(\ell,\left\{p_{k}\right\}_{N}\right)$ is a function of the loop momentum $\ell$ and the $N$ external momenta $\left\{p_{k}\right\}_{N}$. The Feynman propagators $G_{F}\left(q_{i}\right)=\left(q_{i}^{2}-m_{i}^{2}+\imath 0\right)^{-1}$ carry momenta $q_{i}=\ell+k_{i}$, where $k_{i}$ are linear combinations of the external momenta. Applying the loop-tree duality theorem this amplitude is rewritten as

$\mathcal{A}_{N}^{(1)}=-\int_{\ell} \mathcal{N}\left(\ell,\left\{p_{k}\right\}_{N}\right) \sum_{i=1}^{N} \tilde{\delta}\left(q_{i}\right)\left(\prod_{j \neq i} G_{D}\left(q_{i} ; q_{j}\right)\right)$,

where $G_{D}\left(q_{i} ; q_{j}\right)=\left(q_{j}^{2}-m_{j}^{2}-\imath 0 \eta \cdot k_{j i}\right)^{-1}$, with $k_{j i}=$ $q_{j}-q_{i}$, are the so-called dual propagators and $\eta$ is an arbitrary future-like vector. The dual propagators differ from the Feynman propagators only in their infinitesimal imaginary prescription, whose sign in the dual propagator depends on the external momenta. A different internal loop momentum is set on-shell in each of the terms in Eq. (2), which are conventionally called dual amplitudes, through the modified delta functional $\tilde{\delta}\left(q_{i}\right)=2 \pi i \theta\left(q_{i, 0}\right) \delta\left(q_{i}^{2}-m_{i}^{2}\right)$, in short, or $\tilde{\delta}\left(q_{i} ; m_{i}\right) \equiv \tilde{\delta}\left(q_{i}\right)$ whenever it is necessary to make reference to the internal masses. Due to the on-shell conditions, the dimension of the integration domain is reduced by one unit. The choice $\eta=(1, \mathbf{0})$ is the most convenient because it is equivalent to integrating out the energy component of the loop momentum, thus reducing the integration measure to the Euclidean space of the loop three-momentum.

The behaviour of scattering amplitudes is ruled by their analytic properties. Aiming for asymptotic expansions at integrand-level, we must therefore consider in detail the analysis of propagators which are the objects that give rise to singularities. While the numerator plays a role in determining whether the amplitude has a UV divergence this is not relevant for the discussion that follows about asymptotic expansions since within LTD the singular UV behaviour is neutralized through local renormalization before integration. An example of this will be shown in the following section.

The dual propagators can manifest non-causal or unphysical singularities on top of the physical divergences related to causal threshold and IR singularities. These unphysical divergences appear only when the various terms in the sum are considered separately. Identifying the conditions under which both causal and unphysical singularities appear as well as their position in the integration space is necessary groundwork for asymptotically expanding an amplitude. The examination of said singularities can be achieved efficiently by reparametrizing the dual propagators as shown in Refs. [21,33] 


$$
\begin{aligned}
\frac{\tilde{\delta}\left(q_{i}\right)}{\pi \imath} G_{D}\left(q_{i} ; q_{j}\right) & =\frac{\delta\left(q_{i, 0}-q_{i, 0}^{(+)}\right)}{q_{i, 0}^{(+)} \lambda_{i j}^{+-} \lambda_{i j}^{++}}, \\
\lambda_{i j}^{ \pm \pm} & = \pm q_{i, 0}^{(+)} \pm q_{j, 0}^{(+)}+k_{j i, 0},
\end{aligned}
$$

where $q_{s, 0}^{(+)}=\sqrt{\mathbf{q}_{s}^{2}+m_{s}^{2}}$ are the on-shell energies. In this notation, a causal unitarity threshold appears for $\lambda_{i j}^{++} \rightarrow 0$ while an unphysical singularity appears for $\lambda_{i j}^{+-} \rightarrow 0$. The latter case always appears entangled between two dual amplitudes which leads to the cancellation of these unphysical singularities due to the always opposite sign of the infinitesimal imaginary prescription in the dual propagators. It is straightforward to derive the kinematic conditions for either of these limits to occur and examples are provided in Refs. [21,33]. In some special kinematic configurations, the unphysical singularities may even be avoided altogether by redefining the loop momentum flow through $\ell \rightarrow-\ell$, see e.g. Ref. [33]. Remarkably, we have recently presented dual representations of selected multiloop topologies that are explicitly free of unphysical singularities, and we conjectured that this property holds to other loop topologies at all orders [34]. The advantages that the causal representation introduces will be illustrated in Sect. 5.

Having identified the propagators of the amplitude that lead to singularities, we can now reparametrize the dual propagators in the following form that is more suitable for asymptotic expansions

$\tilde{\delta}\left(q_{i}\right) G_{D}\left(q_{i} ; q_{j}\right)=\frac{\tilde{\delta}\left(q_{i}\right)}{2 q_{i} \cdot k_{j i}+\Gamma_{i j}+\Delta_{i j}-\imath 0 \eta \cdot k_{j i}}$,

where $\Gamma_{i j}+\Delta_{i j}=k_{j i}^{2}+m_{i}^{2}-m_{j}^{2}$. If $\Gamma_{i j}+\Delta_{i j}$ vanishes the dual propagator is not expanded. Otherwise the starting point for the asymptotic expansion is to demand that the condition

$\left|\Delta_{i j}\right| \ll\left|2 q_{i} \cdot k_{j i}+\Gamma_{i j}\right|$

be fulfilled for the whole range of the loop integration space except for potentially small regions around physical divergences. The distinctive feature of LTD is that since dual propagators only appear in integrands where one loop momentum has been set on-shell, the condition has to be fulfilled in the Euclidean space of the loop three-momentum. Whenever it is satisfied, the dual propagator can be expanded as

$G_{D}\left(q_{i} ; q_{j}\right)=\sum_{n=0}^{\infty} \frac{\left(-\Delta_{i j}\right)^{n}}{\left(2 q_{i} \cdot k_{j i}+\Gamma_{i j}-i 0 \eta \cdot k_{j i}\right)^{n+1}}$,

or in the case of amplitudes with propagators raised to multiple powers, as often occurs in multiloop amplitudes, by using the generalized binomial theorem

$$
\begin{aligned}
& \left(G_{D}\left(q_{i} ; q_{j}\right)\right)^{m} \\
& \quad=\sum_{n=0}^{\infty}\left(\begin{array}{c}
-m \\
n
\end{array}\right) \frac{\left(\Delta_{i j}\right)^{n}}{\left(2 q_{i} \cdot k_{j i}+\Gamma_{i j}-i 0 \eta \cdot k_{j i}\right)^{n+m}} .
\end{aligned}
$$

A special case of the above is the situation when $k_{j i}^{2}+m_{i}^{2}-m_{j}^{2}$ is much smaller than the scalar product $2 q_{i} \cdot k_{j i}$. Then we must identify $\Gamma_{i j}=0$ and the expansion above simplifies as follows:

$G_{D}\left(q_{i} ; q_{j}\right)=\sum_{n=0}^{\infty} \frac{\left(-\Delta_{i j}\right)^{n}}{\left(2 q_{i} \cdot k_{j i}\right)^{n+1}}$.

The asymptotic expansion of the dual propagators given in Eq. (6) is the basis for the majority of the examples that will be presented in this work. In the following, we will discuss how to select the functions $\Gamma_{i j}$ and $\Delta_{i j}$ in different kinematical limits. Further simplifications arise whenever $\mathbf{k}_{j i}=0$. In that case, with the change of variables $\left|\mathbf{q}_{\mathbf{i}}\right|=m_{i} / 2\left(x_{i}-x_{i}^{-1}\right)$, the denominator of the expanded dual propagator takes an easily integrable form.

For the case of $\Gamma_{i j}=0$ the denominator of Eq. (8) is given by

$2 q_{i} \cdot k_{j i}=k_{j i, 0} m_{i}\left(x_{i}+x_{i}^{-1}\right)$.

Otherwise, the denominator of Eq. (6) can be written as

$$
\begin{aligned}
& 2 q_{i} \cdot k_{j i}+\Gamma_{i j}-\imath 0 \eta \cdot k_{j i} \\
& \quad=Q_{i}^{2}\left(x_{i}+r_{i j}\right)\left(x_{i}^{-1}+r_{i j}\right) .
\end{aligned}
$$

The form found here determines the parameters $\Gamma_{i j}$ and $r_{i j}$ appearing in the expansion to be restricted by the conditions

$$
\begin{aligned}
\Gamma_{i j}-\imath 0 \eta \cdot k_{j i} & =Q_{i}^{2}\left(1+r_{i j}^{2}\right), \\
r_{i j} & =\frac{m_{i} k_{j i, 0}}{Q_{i}^{2}}-\frac{\imath 0 \eta \cdot k_{j i}}{Q_{i}^{2}},
\end{aligned}
$$

assuming $\left|r_{i j}\right| \leq 1$. For the class of limits where one hard scale $Q$ is available, we can identify $Q_{i}^{2}= \pm Q^{2}$, where the sign is determined by the sign of the hard scale in the expression $k_{j i}^{2}+m_{i}^{2}-m_{j}^{2}$. As will be seen in the examples of the following sections this type of expansion facilitates the analytical integration based on integrals of the form

$$
\int_{1}^{\infty} \frac{\mathrm{d} x_{i}}{x_{i}\left(x_{i}+r_{i j}\right)\left(x_{i}^{-1}+r_{i j}\right)}=\frac{\log \left(r_{i j}\right)}{r_{i j}^{2}-1}, \quad\left|r_{i j}\right|<1 .
$$

On top of the relations in Eq. (11) additional conditions are to be respected by the expansion parameters. The expansion is to converge both at integrand- and at integral-level and the analytic behaviour of the dual propagator may not be fundamentally changed. This is to mean that for a propagator with a singularity the expansion is to also display that singularity, while the expansion of a non-singular propagator is to be finite in all of the integration domain as well. The 
infinitesimal imaginary prescription of $r_{i j}$ given in Eq. (11) accounts properly for the complex prescription of the original dual propagator and therefore its causal thresholds. This corresponds to the argument $r_{i j}$ of the logarithm in Eq. (12) taking a negative value, $\operatorname{Re}\left(r_{i j}\right)<0$.

While the scenario described above covers the majority of typical limits, asymptotic expansions at thresholds deserve a special treatment since all the scales are of the same order and, therefore, a hard scale cannot be clearly identified. Even when approaching the physical threshold from below and thus considering a dual propagator without pole on the real axis, its behaviour is still strongly influenced by the threshold singularity. In cases like this it is necessary to consider the trajectory of the pole in the non-expanded propagator more carefully, which is determined by

$x_{i}=-\frac{k_{j i, 0}^{2}+m_{i}^{2}-m_{j}^{2} \pm \lambda^{1 / 2}\left(k_{j i, 0}^{2}, m_{i}^{2}, m_{j}^{2}\right)}{2 k_{j i, 0} m_{i}}$,

in terms of the modified Källén function $\lambda\left(k_{j i, 0}^{2}, m_{i}^{2}, m_{j}^{2}\right)=$ $\left(k_{j i, 0}^{2}-\left(m_{i}+m_{j}\right)^{2}\right)\left(k_{j i, 0}^{2}-\left(m_{i}-m_{j}\right)^{2}\right)-{ }_{l} 0 k_{j i, 0}\left(k_{j i, 0}^{2}+\right.$ $m_{i}^{2}-m_{j}^{2}$ ). Then, by expanding close to threshold

$$
\begin{aligned}
& \left.x_{i}\right|_{\beta \rightarrow 0^{ \pm}} \\
& \quad=-\operatorname{sign}\left(k_{j i, 0}\right)\left(1 \pm \sqrt{-\frac{m_{j} \beta}{m_{i}}-\imath 0 k_{j i, 0}}+\mathcal{O}(\beta)\right),
\end{aligned}
$$

with $\beta$ defined through $k_{j i, 0}^{2}=\left(m_{i}+m_{j}\right)^{2}(1-\beta)$. Following this procedure we can deduce the correct $r_{i j}$ parameters for the asymptotic expansion both from above and from below threshold and bring the dual propagator into the desired form, Eq. (10), while showcasing the same threshold behaviour as the non-expanded propagator.

In the following sections we will apply these general ideas to benchmark one-loop integrals, and will present their asymptotic expansions in several kinematical limits within the LTD formalism.

\section{Asymptotic expansion of the scalar two-point function with two internal masses}

An obvious first benchmark application is the asymptotic expansion of the scalar two-point function with two different internal masses corresponding to the diagram in Fig. 1. The corresponding amplitude in the Feynman representation is

$\mathcal{A}^{(1)}=\int_{\ell} G_{F}\left(q_{1} ; M\right) G_{F}\left(q_{2} ; m\right)$,

with $q_{1}=\ell-p$ and $q_{2}=\ell$. The momentum flow, assuming $p_{0}>0$, has been chosen to avoid the appearance of noncausal or unphysical singularities. These types of integrand

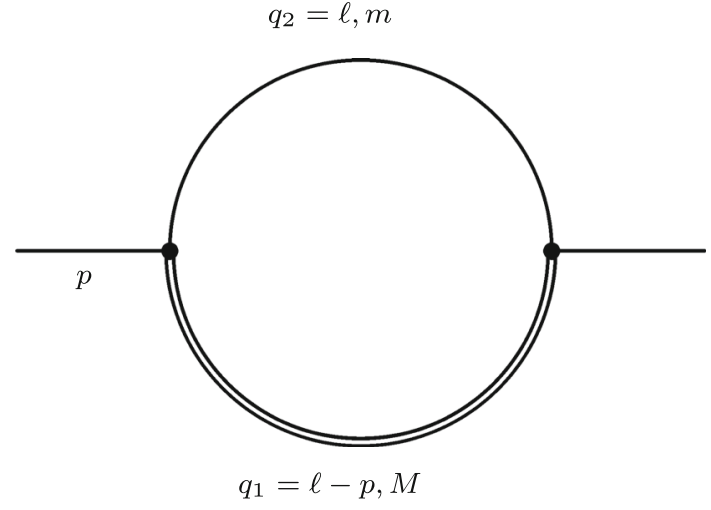

Fig. 1 Two-point function with internal masses $M>m$

singularities, if they appear, always cancel in the sum of dual amplitudes.

We are interested in the asymptotic expansion of the renormalized amplitude, which is well defined directly in four space-time dimensions

$\mathcal{A}^{(1, R)}=\mathcal{A}^{(1)}-\left.\mathcal{A}_{\mathrm{UV}}^{(1)}\right|_{d=4}$,

where $\mathcal{A}_{\mathrm{UV}}^{(1)}$ is a local UV counterterm that suppresses the singular behaviour of the unintegrated amplitude for large loop momenta. ${ }^{1}$ Its Feynman and dual representations are respectively given by

$\mathcal{A}_{\mathrm{UV}}^{(1)}=\int_{\ell}\left(G_{F}\left(\ell ; \mu_{\mathrm{UV}}\right)\right)^{2}=\int_{\ell} \frac{\tilde{\delta}\left(\ell ; \mu_{\mathrm{UV}}\right)}{2\left(\ell_{0, \mathrm{UV}}^{(+)}\right)^{2}}$,

$\ell_{0, \mathrm{UV}}^{(+)}=\sqrt{\ell^{2}+\mu_{\mathrm{UV}}^{2}}$

where $\mu_{\mathrm{UV}}$ is an arbitrary scale. Its integrated form takes the shape

$\mathcal{A}_{\mathrm{UV}}^{(1)}=\frac{\Gamma(1+\epsilon)}{(4 \pi)^{2-\epsilon}} \frac{1}{\epsilon}\left(\frac{\mu_{\mathrm{UV}}^{2}}{\mu^{2}}\right)^{-\epsilon}$,

and implements the standard $\overline{M S}$ renormalization scheme when identifying the parameter $\mu_{\mathrm{UV}}$ with the DREG renormalization scale $\mu$. The full analytic expression of the renormalized scalar two-point function is well known through standard techniques

$$
\begin{aligned}
\mathcal{A}^{(1, R)}= & \frac{1}{16 \pi^{2}}\left[2+\frac{p^{2}+M^{2}-m^{2}}{2 p^{2}} \log \left(\frac{\mu_{\mathrm{UV}}^{2}}{M^{2}}\right)\right. \\
& +\frac{p^{2}+m^{2}-M^{2}}{2 p^{2}} \log \left(\frac{\mu_{\mathrm{UV}}^{2}}{m^{2}}\right)
\end{aligned}
$$

${ }^{1}$ For realistic scattering amplitudes the UV counterterm should contain also contributions that integrate to zero and a finite renormalization to preserve the Ward identities, see e.g. [25]. 


$$
\begin{aligned}
& +\frac{\lambda^{1 / 2}\left(p^{2}, m^{2}, M^{2}\right)}{p^{2}} \\
& \left.\cdot \log \left(\frac{m^{2}+M^{2}-p^{2}+\lambda^{1 / 2}\left(p^{2}, m^{2}, M^{2}\right)}{2 m M}\right)\right],
\end{aligned}
$$

which is symmetric under the exchange $m \leftrightarrow M$. This expression will be used to check the validity of the asymptotic expansions presented in the next sections.

\subsection{Master asymptotic expansion}

The dual representation of the renormalized scalar two-point function (Eq. (16)) is given by

$$
\begin{aligned}
\mathcal{A}^{(1, R)}= & -\int_{\ell}\left[\tilde{\delta}\left(q_{1} ; M\right) G_{D}\left(q_{1} ; \ell\right)+\tilde{\delta}(\ell ; m) G_{D}\left(\ell ; q_{1}\right)\right. \\
& \left.+\frac{1}{2} \tilde{\delta}\left(\ell ; \mu_{\mathrm{UV}}\right)\left(\ell_{0, \mathrm{UV}}^{(+)}\right)^{-2}\right]
\end{aligned}
$$

where the dual propagators are

$$
\begin{aligned}
G_{D}\left(q_{1} ; \ell\right) & =\frac{1}{2 q_{1} \cdot p+p^{2}-m^{2}+M^{2}-\imath 0 p_{0}}, \\
G_{D}\left(\ell ; q_{1}\right) & =\frac{1}{-2 \ell \cdot p+p^{2}+m^{2}-M^{2}+\imath 0 p_{0}} .
\end{aligned}
$$

Setting $p=\left(p_{0}, \mathbf{0}\right)$ with $p_{0}>0$, the on-shell energies and scalar products are $q_{1,0}^{(+)}=\sqrt{\ell^{2}+M^{2}}, \ell_{0}^{(+)}=\sqrt{\ell^{2}+m^{2}}$, $q_{1} \cdot p=q_{1,0}^{(+)} p_{0}$ and $\ell \cdot p=\ell_{0}^{(+)} p_{0}$. With this choice of the reference frame the dual representation and its asymptotic expansion become particularly simple as the angular integration of the loop three-momentum is straight. The renormalized result can be reproduced through direct integration of Eq. (20).

Still, the general propagator expansion of Eqs. (6) and (10) can be applied to this amplitude to simplify the integrand

$$
\begin{aligned}
\mathcal{A}^{(1, R)}= & -\frac{1}{16 \pi^{2}}\left[\sum_{i, j=1,2} \frac{m_{i}^{2}}{Q_{i}^{2}} \sum_{n=0}^{\infty}\left(-\frac{\Delta_{i j}}{Q_{i}^{2}}\right)^{n} I^{(n)}\left(r_{i j}, m_{i}\right)\right. \\
& \left.+I_{\mathrm{UV}}\left(\mu_{\mathrm{UV}}\right)\right]
\end{aligned}
$$

where $m_{1}=M, m_{2}=m$ and the remaining integrals are contained in

$$
\begin{aligned}
& I^{(n)}\left(r_{i j}, m_{i}\right) \\
& \quad=\lim _{\Lambda \rightarrow \infty} \int_{1}^{\frac{\Lambda+\sqrt{\Lambda^{2}+m_{i}^{2}}}{m_{i}}} \mathrm{~d} x \frac{\left(x^{2}-1\right)^{2} x^{-3}}{\left[\left(x+r_{i j}\right)\left(x^{-1}+r_{i j}\right)\right]^{n+1}},
\end{aligned}
$$

and

$$
I_{\mathrm{UV}}\left(\mu_{\mathrm{UV}}\right)=\lim _{\Lambda \rightarrow \infty} \int_{1}^{\frac{\Lambda+\sqrt{\Lambda^{2}+\mu_{\mathrm{UV}}^{2}}}{\mu_{\mathrm{UV}}}} \mathrm{d} x \frac{2\left(x^{2}-1\right)^{2} x^{-1}}{\left(x^{2}+1\right)^{2}} .
$$

We have introduced a cutoff $\Lambda$ because the individual contributions are still singular in the UV. The sum of all of them is UV finite, however. Therefore, we can safely work in four space-time dimensions and then take the limit $\Lambda \rightarrow \infty$ after integration. Notice that the cutoff is a valid regulator because it acts on the Euclidean space of the loop three-momentum. The results of these integrals, up to order $n=2$, are given by

$$
\begin{aligned}
& I^{(n)}\left(r_{i j}, m_{i}\right) \\
& \stackrel{n=0}{=} \lim _{\Lambda \rightarrow \infty}\left[\frac{2 \Lambda}{m_{i} r_{i j}}-\left(1+\frac{1}{r_{i j}^{2}}\right) \log \left(\frac{2 \Lambda}{m_{i}}\right)\right. \\
& \left.\quad+\left(1-\frac{1}{r_{i j}^{2}}\right) \log \left(r_{i j}\right)\right], \\
& \stackrel{n=1}{=} \lim _{\Lambda \rightarrow \infty}\left[-\frac{1}{r_{i j}^{2}}\left(1-\log \left(\frac{2 \Lambda}{m_{i}}\right)-\frac{1+r_{i j}^{2}}{1-r_{i j}^{2}} \log \left(r_{i j}\right)\right)\right], \\
& \stackrel{n=2}{=} \frac{1}{\left(1-r_{i j}^{2}\right)^{2}}\left(\frac{1+r_{i j}^{2}}{2 r_{i j}^{2}}+\frac{2}{1-r_{i j}^{2}} \log \left(r_{i j}\right)\right),
\end{aligned}
$$

and

$$
I_{\mathrm{UV}}\left(\mu_{\mathrm{UV}}\right)=\lim _{\Lambda \rightarrow \infty}\left[2 \log \left(\frac{2 \Lambda}{\mu_{\mathrm{UV}}}\right)-2\right] .
$$

A noteworthy feature of this expansion is that the UV divergence lessens with each order in the expansion. Indeed, all the contributions with $n \geq 2$ are UV finite, and can be calculated directly by extending the upper limit of the integral to infinity. The linearly UV divergent terms appearing at $n=0$ cancel between the two dual amplitudes and the logarithmic dependence on the UV cutoff $\Lambda$ of both terms at $n=0$ and $n=1$ is canceled by the UV counterterm. Since these cancellations happen locally in momentum space, numerical integration in the UV limit is straightforward.

The asymptotic expansion of the renormalized amplitude then takes the general form

$$
\begin{aligned}
\mathcal{A}^{(1, R)}= & \frac{1}{16 \pi^{2}} \sum_{i, j}\left[2+c_{0, i} \log \left(\frac{\mu_{\mathrm{UV}}}{m_{i}}\right)\right. \\
& \left.+\sum_{n=0}^{\infty}\left(c_{1, i}^{(n)}+c_{2, i}^{(n)} \log \left(r_{i j}\right)\right)\right] .
\end{aligned}
$$

The coefficient $c_{i, 0}$ is given by

$$
c_{0, i}=\frac{m_{i}^{2}}{Q_{i}^{2}}\left(1+\frac{1}{r_{i j}^{2}}\left(1+\frac{\Delta_{i j}}{Q_{i}^{2}}\right)\right)=\frac{p^{2}+m_{i}^{2}-m_{j}^{2}}{p^{2}}
$$


Table 1 The coefficients of the asymptotic expansions for the scalar two-point function in different kinematical limits

$M^{2} \gg\left\{m^{2}, p^{2}\right\} \quad p^{2} \gg\left\{m^{2}, M^{2}\right\} \quad p^{2}=(m+M)^{2}(1-\beta), \quad \beta \rightarrow 0^{ \pm}$

$G_{D}\left(q_{1} ; \ell\right)$

$\begin{array}{lll}\Gamma_{12} & M^{2}+p^{2} & p^{2}+M^{2} \\ \Delta_{12} & -m^{2} & -m^{2} \\ r_{12} & \frac{\sqrt{p^{2}}}{M} & \frac{M}{\sqrt{p^{2}}} \\ Q_{1}^{2} & M^{2} & p^{2}\end{array}$

$G_{D}\left(\ell ; q_{1}\right)$

$\begin{array}{ll}\Gamma_{21} & -M^{2}-\frac{m^{2} p^{2}}{M^{2}} \\ \Delta_{21} & p^{2}+m^{2}+\frac{m^{2} p^{2}}{M^{2}} \\ r_{21} & \frac{m \sqrt{p^{2}}}{M^{2}} \\ Q_{2}^{2} & -M^{2}\end{array}$

$p^{2}+m^{2}$

$-M^{2}$

$-\frac{m}{\sqrt{p^{2}}}+{ }^{2} 0$

$p^{2}$

$$
\begin{aligned}
& 2 M p \cosh \left(\sqrt{-\frac{m \beta}{M}-\imath 0}\right) \\
& p^{2}+M^{2}-m^{2}-\Gamma_{12} \\
& \exp \left(\sqrt{-\frac{m \beta}{M}-\imath 0}\right) \\
& M p \exp \left(-\sqrt{-\frac{m \beta}{M}-\imath 0}\right)
\end{aligned}
$$

$2 m p \cosh \left(\sqrt{-\frac{M \beta}{m}+\imath 0}\right)$

$p^{2}+m^{2}-M^{2}-\Gamma_{21}$

$-\exp \left(\sqrt{-\frac{M \beta}{m}+\imath 0}\right)$

$m p \exp \left(-\sqrt{-\frac{M \beta}{m}+{ }^{\prime 0} 0}\right)$ and the coefficients $c_{1, i}^{(n)}$ and $c_{2, i}^{(n)}$ needed for the first few orders of the expansion are given by

$$
\begin{aligned}
c_{1, i}^{(n)}= & -\frac{m_{i}^{2}}{Q_{i}^{2}}\left\{0, \frac{-\Delta_{i j}}{Q_{i}^{2}} \frac{-1}{r_{i j}^{2}},\left(\frac{-\Delta_{i j}}{Q_{i}^{2}}\right)^{2} \frac{1+r_{i j}^{2}}{2 r_{i j}^{2}\left(1-r_{i j}^{2}\right)^{2}},\right. \\
& \left.\left(\frac{-\Delta_{i j}}{Q_{i}^{2}}\right)^{3} \frac{1+10 r_{i j}^{2}+r_{i j}^{4}}{6 r_{i j}^{2}\left(1-r_{i j}^{2}\right)^{4}}\right\}, \\
c_{2, i}^{(n)}= & -\frac{m_{i}^{2}}{Q_{i}^{2}}\left\{1-\frac{1}{r_{i j}^{2}}, \frac{-\Delta_{i j}}{Q_{i}^{2}} \frac{1+r_{i j}^{2}}{r_{i j}^{2}\left(1-r_{i j}^{2}\right)},\right. \\
& \left.\left(\frac{-\Delta_{i j}}{Q_{i}^{2}}\right)^{2} \frac{2}{\left(1-r_{i j}^{2}\right)^{3}},\left(\frac{-\Delta_{i j}}{Q_{i}^{2}}\right)^{3} \frac{2\left(1+r_{i j}^{2}\right)}{\left(1-r_{i j}^{2}\right)^{5}}\right\} .
\end{aligned}
$$

Each term of the expansion is suppressed by extra powers of $\Delta_{i j}$.

\subsection{Asymptotic expansion for different kinematical limits}

We now consider explicitly different kinematical limits and the corresponding asymptotic expansions. In the limit of one large mass, $M^{2} \gg\left\{m^{2}, p^{2}\right\}$, the expansion parameters are $Q_{1}^{2}=-Q_{2}^{2}=M^{2}, r_{12}=\sqrt{p^{2}} / M$, and $r_{21}=m \sqrt{p^{2}} / M^{2}$. The functions $\Gamma_{i j}$ and $\Delta_{i j}$ are summarized in Table 1.

The election of the expansion parameters in the limit of a large external momentum, $p^{2} \gg\left\{m^{2}, M^{2}\right\}$, is also summarized in Table 1. Since this kinematical configuration is above threshold, the asymptotic expansion should feature an imaginary part just as the original integral. This imaginary part is generated through $\log \left(r_{21}\right)$, with $r_{21}=-m / \sqrt{p^{2}}+\imath 0$.
In the slightly more involved case of the threshold limit with $\beta=1-p^{2} /(m+M)^{2} \rightarrow 0^{ \pm}$, the election of the expansion parameters, also summarized in Table 1, is such that $\log \left(r_{12}\right)=\sqrt{m / M} \sqrt{-\beta-\imath 0}$ and $\log \left(r_{21}\right)=$ $\sqrt{M / m} \sqrt{-\beta+\imath 0}-\imath \pi$. This compact result is obtained by exponentiating the expanded expression determining the position of the threshold in the complex plane given by Eq. (14). Note that going beyond the formalism described in Sect. 2 through exponentiation is not necessary for obtaining a convergent expression but it facilitates a simpler and more intuitive understanding of the expansion. The expressions for $\Delta_{i j}$ and $Q_{i}^{2}$ fulfill the necessary asymptotic behaviour as

$\frac{m_{i}^{2}}{Q_{i}^{2}}=\frac{m_{i}}{m+M}+\mathcal{O}\left(\beta^{1 / 2}\right), \quad \frac{\Delta_{i j}}{Q_{i}^{2}}=\mathcal{O}\left(\beta^{2}\right)$.

The first dual propagator $G_{D}\left(q_{1} ; \ell\right)$ is free of threshold singularities and thus leads to a real expansion independently of the sign of $\beta$. The expressions obtained for both $r_{12}$ and $r_{21}$ can be used both when approaching the threshold from below and from above because the infinitesimal imaginary component accompanying $\beta$ is fixed by the complex prescription of the dual propagators. While in the case below threshold no singularity appears in the propagator on the real axis, it does exist in the complex plane and approaches the path of integration as can be seen in Fig. 2.

In all the kinematical regions studied, we have achieved their asymptotic expansions by conveniently selecting the expansion parameters that are used in a common expression, Eq. (28), that describes all these limits at once. In each limit fast convergence was achieved both at integrand- and at integral-level. 


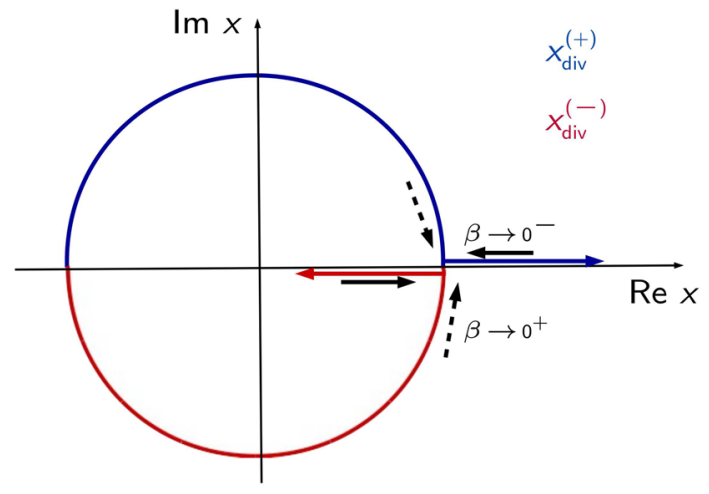

Fig. 2 The position of the singularities of the unexpanded dual propagator in terms of the threshold parameter $\beta=1-\frac{p^{2}}{(m+M)^{2}}$ after the change of variable $\left|\mathbf{q}_{\mathbf{i}}\right|=m_{i} / 2\left(x_{i}-x_{i}^{-1}\right)$. The path of integration goes from the point where the singularities reach the real axis until infinity

\subsection{Comparison with Expansion by Regions}

It is of interest to see how the expansions developed above hold up in comparison to the established method of Expansion by Regions (EbR) [10-17]. Within this successful method the integrand of the Feynman amplitude, written in terms of Minkowski momenta, is expanded by dividing the space of the loop momenta into distinct regions. In each region, the integrand is expanded into a Taylor series with respect to the parameters considered small therein. Consecutively, the expanded integrands are integrated over the whole integration domain, not just within the region where the expansion was justified. The scaleless integrals that may appear are set to zero as commonly done within DREG. While Expansion by Regions has been successful for many types of amplitudes a general proof is still pending. One may raise a few issues with the procedure above which will be mentioned in the context of its application to the scalar twopoint function in Eq. (1). We centre the discussion on the limit of one large mass, $M^{2} \gg\left\{m^{2}, p^{2}\right\}$.

While in a general loop integral many types of regions can appear in this particular example there are only two regions, the hard region with $\ell \sim M$ and the soft region with $\ell \sim\left\{m, \sqrt{p^{2}}\right\}^{2}$. The scalar product between the loop momentum and the external momentum inherits the scaling of the momentum itself, that is for the hard region one performs the replacements

$\left\{\ell^{2}, M^{2}\right\} \rightarrow \lambda^{2}\left\{\ell^{2}, M^{2}\right\}, \quad p \cdot \ell \rightarrow \lambda p \cdot \ell$,

and expands for $\lambda \rightarrow \infty$. The assumed relationship between the large loop momentum and both its square and its scalar products does not account for cancellations between the

${ }^{2}$ To be precise, the scaling is assumed for the momentum in the Euclidean sense, i.e. $|\ell|=\sqrt{\ell_{0}^{2}+\ell^{2}}$. energy component and the spatial components which will take place when integrating over the unrestricted components of the loop momentum. The Taylor series with the prescriptions above and comparable ones for the soft region leads to the expanded integrands

$$
\begin{aligned}
\mathcal{A}_{\text {hard }}^{(1)}= & \int_{\ell}\left(\frac{1}{\ell^{2}}+\frac{m^{2}}{\left(\ell^{2}\right)^{2}}+\frac{m^{4}}{\left(\ell^{2}\right)^{3}}+\ldots\right) \\
& \cdot\left(\frac{1}{\ell^{2}-M^{2}}+\frac{2 p \cdot \ell-p^{2}}{\left(\ell^{2}-M^{2}\right)^{2}}+\frac{4(p \cdot \ell)^{2}+p^{2}}{\left(\ell^{2}-M^{2}\right)^{3}}+\ldots\right), \\
\mathcal{A}_{\mathrm{soft}}^{(1)}= & \int_{\ell} \frac{1}{\ell^{2}-m^{2}} \\
& \cdot\left(-\frac{1}{M^{2}}-\frac{(p-\ell)^{2}}{M^{4}}-\frac{(p-\ell)^{4}}{M^{6}}+\ldots\right) .
\end{aligned}
$$

The first order of the expansion at integrated level is achieved by combining the UV counterterm with the first term appearing in the hard region

$$
\int_{\ell} \frac{1}{\ell^{2}\left(\ell^{2}-M^{2}\right)}-\mathcal{A}_{\mathrm{UV}}^{(1)}=\frac{1-\log \left(\frac{M^{2}}{\mu_{\mathrm{UV}}^{2}}\right)}{16 \pi^{2}}+\mathcal{O}(\epsilon) .
$$

The soft region does not contribute at this order. For the next order one must select all terms in the expansion at integrandlevel which will lead to contributions of order $M^{-2}$. This includes the first term of the expansion in the soft region and three terms from the hard region. The result achieved in this way is indeed the Taylor series $(\mathcal{T})$ of the full result

$$
\begin{aligned}
\mathcal{T} \mathcal{A}^{(1, R)}(M, \infty)= & \frac{1}{16 \pi^{2}}\left(1-\log \left(\frac{M^{2}}{\mu_{\mathrm{UV}}^{2}}\right)\right. \\
& \left.+\frac{p^{2}-2 m^{2} \log \left(\frac{M^{2}}{m^{2}}\right)}{2 M^{2}}+\ldots\right) .
\end{aligned}
$$

In direct comparison, we give here the first renormalized orders of the series achieved through the general expansion of the dual propagator Eq. (28) in the limit of one large mass:

$$
\begin{aligned}
\mathcal{A}_{n \leq 1}^{(1, R)}= & \frac{1}{16 \pi^{2}}\left(1-\log \left(\frac{M^{2}}{\mu_{\mathrm{UV}}^{2}}\right)-\frac{m^{2}}{M^{2}}\right. \\
& -\frac{m^{2}\left(M^{2}+m^{2}+p^{2}\right)}{M^{4}-m^{2} p^{2}} \log \left(\frac{M^{2}}{m^{2}}\right) \\
& \left.+\frac{m^{2}\left(\left(p^{2}\right)^{2}-m^{2} M^{2}\right)}{\left(M^{2}-p^{2}\right)\left(M^{4}-m^{2} p^{2}\right)} \log \left(\frac{M^{2}}{p^{2}}\right)\right) .
\end{aligned}
$$

By including the next term of the expansion $(n=2)$ and then expanding the rational coefficients for $M^{2} \gg\left\{m^{2}, p^{2}\right\}$, we recover the expected Taylor series. Higher terms of the Taylor series can be obtained by including more terms in the dual 
Table 2 The relative errors with respect to the full result of the dual expansion as given from Eq. (28) (both the direct result of this expansion and considering only the leading behaviour at large $M$ ) compared with the result obtained through Expansion by Regions. Numerical evaluation with parameters $M=10 m, p^{2}=3 m^{2}$, and $\mu_{\mathrm{UV}}=M$

\begin{tabular}{llllll}
\hline & $G_{D}$ expansion & $\mathcal{O}\left(M^{-2}\right)$ & $\mathcal{O}\left(M^{-4}\right)$ & EbR \\
\hline$n=1$ & $2.67 \%$ & $2.45 \%$ & $2.68 \%$ & $\mathcal{O}\left(M^{-0}\right)$ & $3.34 \%$ \\
$n=2$ & $0.0375 \%$ & $0.135 \%$ & $0.0300 \%$ & $\mathcal{O}\left(M^{-2}\right)$ & $0.135 \%$ \\
$n=3$ & $7.60 \cdot 10^{-6}$ & $0.135 \%$ & $6.18 \times 10^{-5}$ & $\mathcal{O}\left(M^{-4}\right)$ & $6.18 \times 10^{-5}$ \\
\hline
\end{tabular}

expansion, $n \geq 3$. The asymptotic expansions in Eqs. (36) and (37) display the same logarithmic dependence, although they differ in the rational coefficients accompanying the logs, which partially encode higher orders in the expansion. This is due to the fact that $\Delta_{12}$ includes subleading terms. The expression in Eq. (37) also contains a logarithmic dependence on $\log \left(M^{2} / p^{2}\right)$, which is formally one order higher than Eq. (36) and cancels when more terms in the dual expansion are included.

The relative error obtained by the two expansions with respect to the full result (19) is numerically of the same order of magnitude. For the values of $M=10 m, p^{2}=3 m^{2}$ and $\mu_{\mathrm{UV}}=M$ the relative error obtained including only the leading term in EbR is $3.3 \%$ compared to the $2.7 \%$ obtained by expanding the dual propagator as described above. Including one more order in the expansion the results are given by $0.14 \%$ and $0.038 \%$, respectively. Interestingly, the numerical error at leading order of the expansion of the dual propagators can be reduced by expanding the rational coefficients. The comparison of the results obtained in EbR with those based on the expansion of the dual propagator is summarized in Table 2, demonstrating that the results of EbR can be exactly reproduced through the expansion of the coefficients whenever sufficient terms in the dual expansion have been included.

There is a distinction in the application of the two methods which we would like to emphasize. In EbR it is essential to consider the terms of the expansion at integrand-level to pick out only those which will contribute at a given order of the result. Failing to do so does not only lead to numerical differences but will generally lead to divergent results. This is due to the cancellation between UV and IR singularities appearing in the expansions of the soft and hard region. In contrast, UV renormalization within the method of expanding the dual propagators only involves the lowest orders of the integrand-level expansion. Including higher terms is optional for improving numerical precision and for this purpose it is possible to straight-forwardly include any amount of terms without needing to worry about ensuring cancellations between separate regions.

\subsection{Asymptotic expansion by dual regions}

The properties of dual amplitudes can also be exploited in a more direct way to facilitate asymptotic expansions. After applying LTD to the integrand of a Feynman integral the loop momentum is restricted to on-shell values. Thus, the direct expansion of the integrand into a Taylor series with respect to whichever scale is considered to be small or large is unambiguous. These asymptotic expansions can be done anywhere within the integration domain and depend on the size of the Euclidean loop three-momentum. For example, in the case of the two-point function in the limit of one large mass, $M^{2} \gg\left\{m^{2}, p^{2}\right\}$, two regions in the loop three-momentum can be distinguished. One soft region with $\ell^{2} \ll M^{2}$, and one hard region with $\ell^{2} \gg\left\{m^{2}, p^{2}\right\}$ and $\ell^{2} \sim M^{2}$. We call these regions in the loop three-momentum dual regions because they become accessible only after obtaining a Euclidean integration domain through the application of LTD. The Euclidean integration domain can then be split up into two well-defined integrand-level expansions as

$$
\begin{aligned}
\mathcal{A}^{(1, R)} & =\int_{0}^{\infty} \mathrm{d}|\ell| a(\ell) \\
& =\int_{0}^{\lambda} \mathrm{d}|\ell| \mathcal{T} a(M, \infty)+\int_{\lambda}^{\infty} \mathrm{d}|\ell| \mathcal{T} a(\{\ell, M\}, \infty),
\end{aligned}
$$

where $a(\ell)$ is the unintegrated form of the renormalized amplitude defined in Eq. (16) and $m<\lambda<M$. The integrand-level convergence and the behaviour around the matching scale $\lambda$ is shown in Fig. 3.

Integration of this type of expansion is straight-forward, as the integrand simplifies significantly. Including only the first order of the Taylor expansion in the soft region one obtains the result

$$
\begin{aligned}
\mathcal{A}_{\mathrm{soft}, n=0}^{(1 ; R)} & =\int_{0}^{\lambda} \mathrm{d}|\ell| \mathcal{T}_{0} a(M, \infty) \\
& =\frac{-2}{16 \pi^{2}}\left(\frac{\lambda^{3}}{M^{3}}+\frac{m^{2}}{M^{2}} \operatorname{Sinh}\left(\frac{\lambda}{m}\right)^{-1}-\frac{\lambda m}{M^{2}} \sqrt{1+\frac{\lambda^{2}}{m^{2}}}\right)
\end{aligned}
$$




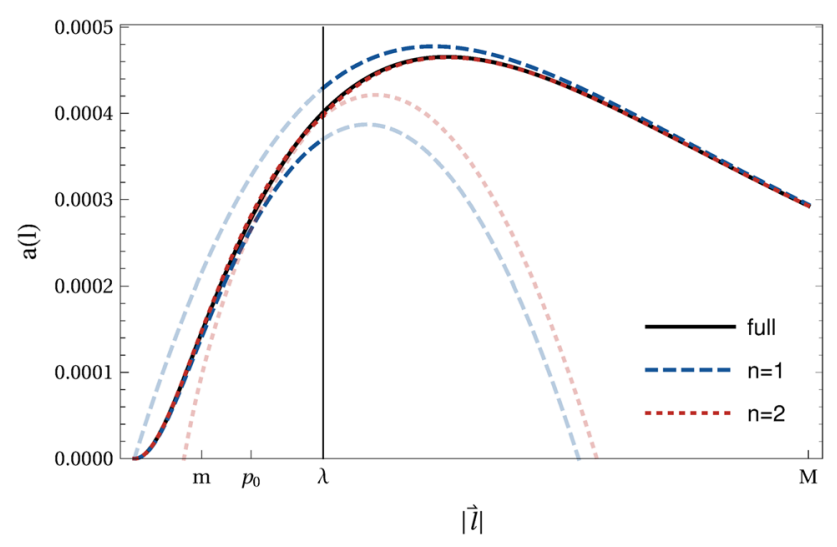

Fig. 3 The convergence at integrand-level of the expansion given in Eq. (38) for the values $M=10 m, p^{2}=3 m^{2}$, and $\mu_{\mathrm{UV}}=M$

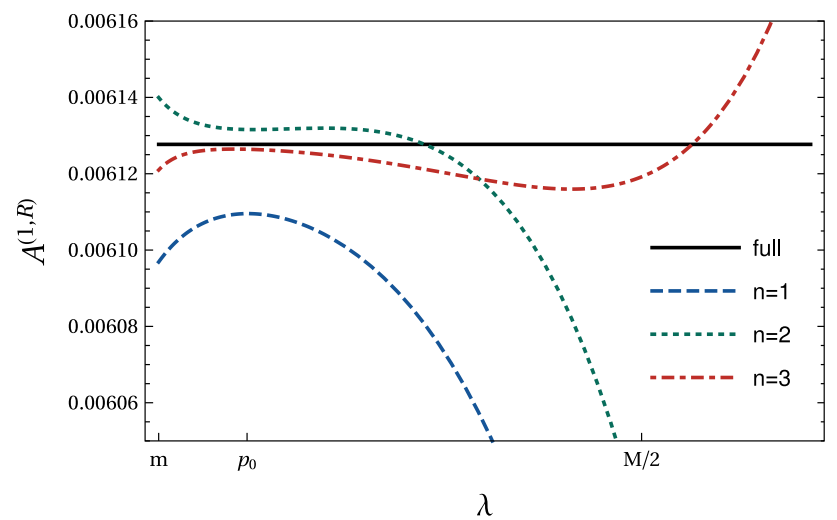

Fig. 4 The convergence of the integrated results of the expansion given in Eq. (38) for the values $M=10 m, p^{2}=3 m^{2}$, and $\mu_{\mathrm{UV}}=M$

while in the hard region

$$
\begin{aligned}
\mathcal{A}_{\text {hard }, n=0}^{(1 ; R)} & =\int_{\lambda}^{\infty} \mathrm{d}|\ell| \mathcal{T}_{0} a(\{\ell, M\}, \infty) \\
& =\frac{1}{16 \pi^{2}}\left(1-\frac{2 \lambda^{2}}{M^{2}}\left(1-\frac{1}{\sqrt{1+\frac{M^{2}}{\lambda^{2}}}}\right)\right),
\end{aligned}
$$

While the soft and hard regions are independent of each other, and the local renormalization is guaranteed in any case, a comparable accuracy in both regions requires to combine the $(n+1)$-th term of the hard region with the $n$-th term of the soft region. We consider this combination of expansion orders in the soft and hard region to be the $n$-th term of the overall expansion. In Fig. 4, we show the result of the asymptotic expansion in Eq. (38) as a function of the matching scale $\lambda$ at different orders. We achieve good order-to-order convergence at the integrated level of the expansion for a wide range of values of $\lambda$.

As would be expected evaluation of the integrated result at the edges of the allowed range for $\lambda$ does not lead to ideal agreement with the full result. The range of values of $\lambda$ for which the amplitude is well approximated increases with rising order in the expansion. An example of this can be seen in Fig. 4. For any order, an appropriate point of evaluation can be obtained by setting the derivative with respect to $\lambda$ and determining the position of the extrema. Coincidentally, the values obtained thus lie very close to $\lambda=p_{0}$. Numerical results for different choices of $\lambda$ are presented in Table 3 .

The main advantage of this Taylor series inspired expansion method is its easy application and automatization.

\section{Asymptotic expansion of the scalar three-point function}

As a benchmark application with more external legs, we consider the scalar three-point function at one-loop as shown in Fig. 5 with all the internal masses equal

$\mathcal{A}_{3}^{(1)}=\int_{\ell} G_{F}\left(q_{1}, q_{2}, q_{3} ; M\right)$,

where $G_{F}\left(q_{1}, q_{2}, q_{3} ; M\right)=\prod_{i=1}^{3} G_{F}\left(q_{i}, M\right)$, with $q_{1}=$ $\ell+p_{1}, q_{2}=\ell+p_{1}+p_{2}$ and $q_{3}=\ell$. Applying LTD to this integral leads to

$$
\begin{aligned}
\mathcal{A}_{3}^{(1)}= & -\int_{\ell}\left[\tilde{\delta}\left(q_{1}\right) G_{D}\left(q_{1} ; q_{2}, q_{3}\right)\right. \\
& \left.+\tilde{\delta}\left(q_{2}\right) G_{D}\left(q_{2} ; q_{1}, q_{3}\right)+\tilde{\delta}\left(q_{3}\right) G_{D}\left(q_{3} ; q_{1}, q_{2}\right)\right],
\end{aligned}
$$

with $G_{D}\left(q_{i} ; q_{j}, q_{k}\right)=G_{D}\left(q_{i} ; q_{j}\right) G_{D}\left(q_{i} ; q_{k}\right)$ based on the dual propagators given as in Eq. (4). The three different linear combinations of external momenta that appear in the dual propagators are $k_{12}=-k_{21}=-p_{2}, k_{13}=-k_{31}=p_{1}$, and $k_{23}=-k_{32}=p_{1}+p_{2}$. Only one of these can be chosen to have a vanishing three-momentum, for example by using the center-of-mass system of $p_{1}$ and $p_{2}$, therefore $\mathbf{p}_{12}=0$. The complete dual integrand thus only has angular dependence in the scalar products $q_{i} \cdot p_{1}$ and $q_{i} \cdot p_{2}$.

Assuming that all the internal particles running in the loop have the same mass $M$ and the external particles are massless $\left(p_{1}^{2}=p_{2}^{2}=0\right.$ and $p_{12}^{2}=s_{12}$, with $\left.p_{12,0}>0\right)$ the LTD representation condenses to

$$
\begin{aligned}
\mathcal{A}_{3}^{(1)}= & -\int_{\ell}\left\{-\frac{\tilde{\delta}\left(q_{1} ; M\right)}{2 q_{1} \cdot p_{12}}\left(\frac{1}{2 q_{1} \cdot p_{1}}+\frac{1}{2 q_{1} \cdot p_{2}}\right)\right. \\
& +\frac{\tilde{\delta}(\ell ; M)}{\left(-2 \ell \cdot p_{2}\right)\left(-2 \ell \cdot p_{12}+s_{12}+\imath 0\right)} \\
& \left.+\frac{\tilde{\delta}(\ell ; M)}{\left(2 \ell \cdot p_{1}\right)\left(2 \ell \cdot p_{12}+s_{12}\right)}\right\},
\end{aligned}
$$


Table 3 Integrated results of the expansion in Eq. (38) evaluated at $\lambda=m, \lambda=3 M / 4$ and $\lambda=p_{0}$, with parameters $M=10 m, p^{2}=3 m^{2}$, and $\mu_{\mathrm{UV}}=M$, compared to the full result of $\mathcal{A}^{(1, R)}=0.006128$

\begin{tabular}{llllll}
\hline Order & $\mathcal{A}^{(1, R)}(\lambda=m)$ & Rel. error & $\mathcal{A}^{(1, R)}(\lambda=3 M / 4)$ & Rel. error & $\mathcal{A}^{(1, R)}\left(\lambda=p_{0}\right)$ \\
\hline 1 & 0.006097 & $0.50 \%$ & 0.004803 & $24 \%$ & 0.006110 \\
2 & 0.006140 & $0.20 \%$ & 0.005100 & $18 \%$ & 0.006132 \\
3 & 0.006121 & $0.11 \%$ & 0.006473 & $5.5 \%$ & 0.006126 \\
\hline
\end{tabular}

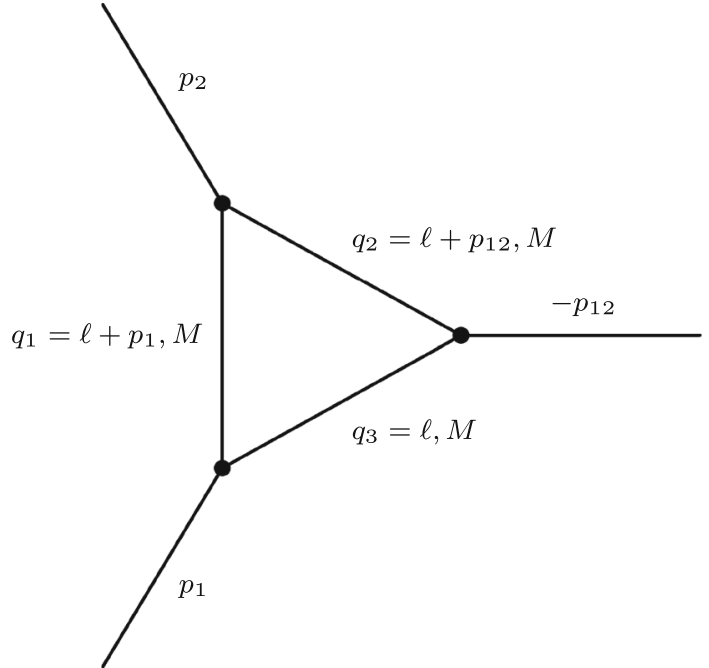

Fig. 5 The three-point function with equal internal masses

with the on-shell energies $q_{1,0}^{(+)}=\sqrt{\left(\boldsymbol{\ell}+\mathbf{p}_{1}\right)^{2}+M^{2}}$ and $q_{2,0}^{(+)}=q_{3,0}^{(+)}=\ell_{0}^{(+)}=\sqrt{\ell^{2}+M^{2}}$. Thus, the on-shell energies in the second and third on-shell cuts are identical.

We may use the following integral identity

$\int_{\ell} \frac{\tilde{\delta}\left(q_{1} ; M\right)}{\left(2 q_{1} \cdot p_{12}\right)\left(2 q_{1} \cdot p_{i}\right)}=\int_{\ell} \frac{\tilde{\delta}(\ell ; M)}{\left(2 \ell \cdot p_{12}\right)\left(2 \ell \cdot p_{i}\right)}$,

to consequently rewrite Eq. (43) as

$$
\begin{aligned}
\mathcal{A}_{3}^{(1)}= & \int_{\ell} \frac{\tilde{\delta}(\ell ; M) s_{12}}{\left(2 \ell \cdot p_{12}\right)\left(2 \ell \cdot p_{1}\right)}\left\{\frac{1}{-2 \ell \cdot p_{12}+s_{12}+\imath 0}\right. \\
& \left.+\frac{1}{2 \ell \cdot p_{12}+s_{12}}\right\} .
\end{aligned}
$$

Notice that now both terms in this expression are UV finite. Therefore, they can be integrated the loop three-momentum separately without the necessity of introducing a cut-off.

The loop three-momentum can be parametrized as

$\ell=|\ell|\left(2 \sqrt{v(1-v)} \hat{e}_{\perp}, 1-2 v\right)$

where $\hat{e}_{\perp}$ is the unit vector perpendicular to $\mathbf{p}_{1}$. The angular dependence then takes the shape

$2 \ell \cdot p_{i}=\sqrt{s_{12}}\left(\ell_{0}^{(+)} \mp|\ell|(1-2 v)\right), \quad i=1,2$.
In this expression the two angular integrations are related by the change of variables $v \rightarrow 1-v$ and thus

$$
\int_{0}^{1} \frac{d v}{2 \ell \cdot p_{i}}=\frac{1}{2 \sqrt{s_{12}}|\ell|} \log \left(\frac{\ell_{0}^{(+)}+|\ell|}{\ell_{0}^{(+)}-|\ell|}\right), \quad i=1,2,
$$

where the usual change of variables $|\ell|=M / 2\left(x-x^{-1}\right)$ with $x>1$ can be employed to obtain the full analytic result

$$
\begin{aligned}
\mathcal{A}_{3}^{(1)}= & \frac{1}{32 \pi^{2} s_{12}} \\
& \times \log ^{2}\left(\frac{\sqrt{s_{12}\left(s_{12}-4 M^{2}\right)}+2 M^{2}-s_{12}}{2 M^{2}}\right) .
\end{aligned}
$$

The large mass expansion is straightforward and it is free of thresholds, i.e. the $\iota 0$ prescription can be dropped when $r=s_{12} / M^{2} \ll 1$. We need to consider both $G_{D}\left(q_{2} ; q_{3}\right)$ and $G_{D}\left(q_{3} ; q_{2}\right)$ in the context of the general propagator expansion. Since in both propagators the condition $\Gamma+\Delta=s_{12}<$ $M \sqrt{s_{12}}$ holds we must identify $\Gamma=0$ and $\Delta=s_{12}$. Thus the asymptotic expansion of the propagators are given by

$G_{D}\left(q_{2} ; q_{3}\right)=\frac{1}{-2 q_{2} \cdot p_{12}+s_{12}}=\sum_{n=0}^{\infty} \frac{\left(-s_{12}\right)^{n}}{\left(-2 q_{2} \cdot p_{12}\right)^{n+1}}$

and

$G_{D}\left(q_{3} ; q_{2}\right)=\frac{1}{2 q_{3} \cdot p_{12}+s_{12}}=\sum_{n=0}^{\infty} \frac{\left(-s_{12}\right)^{n}}{\left(2 q_{3} \cdot p_{12}\right)^{n+1}}$.

Combining the two expanded propagators one obtains a single asymptotic expansion as

$G_{D}\left(q_{2} ; q_{3}\right)+G_{D}\left(q_{3} ; q_{2}\right)=-\frac{2}{s_{12}} \sum_{n=1}^{\infty}\left(\frac{s_{12}}{2 \ell \cdot p_{12}}\right)^{2 n}$,

leading to the expanded amplitude

$$
\begin{aligned}
& \mathcal{A}_{3}^{(1)}\left(s_{12} \ll M^{2}\right) \\
& =-\int_{\ell} \frac{\tilde{\delta}(\ell ; M)}{\left(2 \ell \cdot p_{12}\right)\left(\ell \cdot p_{1}\right)} \sum_{n=1}\left(\frac{s_{12}}{2 \ell \cdot p_{12}}\right)^{2 n} .
\end{aligned}
$$


Integration leads to the following result for the large mass expansion:

$$
\begin{aligned}
& \mathcal{A}_{3}^{(1)}\left(s_{12} \ll M^{2}\right) \\
& =-\frac{1}{16 \pi^{2}} \frac{1}{2 M^{2}}\left(1+\frac{r}{12}+\frac{r^{2}}{90}\right)+\mathcal{O}\left(r^{3}\right) .
\end{aligned}
$$

For $M / \sqrt{s_{12}}=3$ the relative error of the result is $9 \times 10^{-3}$ with only the first term of the expansion and reduces to $1 \times$ $10^{-4}$ and $2 \times 10^{-6}$ when including up the second and third term of the expansion, respectively.

In the small mass limit, $2 M / \sqrt{s_{12}} \ll 1$, the general expansion of the dual propagators can be applied as well. The expansion parameters are

$$
\begin{aligned}
& \Gamma_{32}=\Gamma_{23} \equiv \Gamma=s_{12}\left(1+\frac{M^{2}}{s_{12}}\right), \\
& r_{32}=-r_{23}=-\frac{M}{\sqrt{s_{12}}}+\iota 0, \\
& Q_{2}^{2}=Q_{3}^{2}=s_{12} .
\end{aligned}
$$

This leads to the expanded amplitude

$$
\begin{aligned}
\mathcal{A}_{3}^{(1)}\left(s_{12} \gg M^{2}\right)= & \int_{\ell} \frac{\tilde{\delta}(\ell ; M) s_{12}}{\left(2 \ell \cdot p_{12}\right)\left(2 \ell \cdot p_{1}\right)} \\
& \cdot \sum_{n=0}^{\infty}\left\{\frac{M^{2 n}}{\left(-2 \ell \cdot p_{12}+\Gamma\right)^{n+1}}\right. \\
& \left.+\frac{M^{2 n}}{\left(2 \ell \cdot p_{12}+\Gamma\right)^{n+1}}\right\} .
\end{aligned}
$$

Alternatively, both propagators in the sum may be combined as

$\mathcal{A}_{3}^{(1)}=\int_{\ell} \frac{\tilde{\delta}(\ell ; M)}{\left(2 \ell \cdot p_{12}\right)\left(2 \ell \cdot p_{1}\right)} \frac{2 s_{12}^{2}}{\left(-\left(2 \ell \cdot p_{12}\right)^{2}+s_{12}^{2}+\imath 0\right)}$,

and expanded similarly to the general expansion so as to obtain the final expanded form at integrand level

$$
\begin{aligned}
\mathcal{A}_{3}^{(1)}\left(s_{12} \gg M^{2}\right)= & \int_{\ell} \frac{\tilde{\delta}(\ell ; M) s_{12}^{2}}{\left(2 \ell \cdot p_{12}\right)\left(\ell \cdot p_{1}\right)} \\
& \cdot \sum_{n=0}^{\infty} \frac{\left(s_{12}^{2} r_{23}^{2}\left(2+r_{23}^{2}\right)\right)^{n}}{\left(-\left(2 \ell \cdot p_{12}\right)^{2}+\Gamma^{2}\right)^{n+1}} .
\end{aligned}
$$

Also in this variation of the expansion it is possible to simplify the denominator in terms of the integration variable $x$ by making use of

$$
\begin{aligned}
& -\left(2 \ell \cdot p_{12}\right)^{2}+s_{12}^{2}\left(1+r_{23}^{2}\right)^{2} \\
& =s_{12}^{2}\left(x^{2}-r_{23}^{2}\right)\left(x^{-2}-r_{23}^{2}\right) .
\end{aligned}
$$

Analytic integration up to $n=1$ gives the result

$$
\begin{aligned}
\mathcal{A}_{3}^{(1)}\left(s_{12} \gg M^{2}\right)= & \frac{1}{16 \pi^{2}} \frac{1}{2\left(1+r_{23}^{2}\right)^{2} s_{12}}\left(\log ^{2}\left(-r_{23}^{2}\right)\right. \\
& +\frac{r_{23}^{2}\left(r_{23}^{2}+2\right) \log \left(-r_{23}^{2}\right)}{1+r_{23}^{2}} \\
& \times\left(\frac{2}{1-r_{23}^{2}}+\frac{\log \left(-r_{23}^{2}\right)}{1+r_{23}^{2}}\right) \\
& \left.+\mathcal{O}\left(r_{23}^{4}\right)\right) .
\end{aligned}
$$

Using the numerical values $\sqrt{s_{12}} / M=3$ the relative error of this result is $33 \%$ (7.5\%) in the real (imaginary) part including only the first term of the expansion and reduces to $7.5 \%$ $(0.04 \%)$ and $1.5 \%(0.26 \%)$ when including up the second and third term of the expansion, respectively. The relative errors obtained by integrating Eq. (56) are slightly better but of the same order of magnitude with $26 \%$ (2.7\%) with only the first term and $3.0 \%(1.3 \%)$ and $0.31 \%(0.27 \%)$ when including up the second and third term of the expansion, respectively. Even better results can be obtained by obtaining the parameters through expansion of the singularity position of the full propagator as discussed in Eq. (14). In this case already at first order the relative error is at $3.9 \%(0.83 \%)$.

\section{Asymptotic expansion of multiloop integrals from the causal LTD representation}

A new LTD representation of multiloop amplitudes has been presented recently [34], which is manifestly causal and, therefore, free of the unphysical singularities discussed in Eq. (3). We will focus in this section on the class of multiloop integrals known as Maximal-Loop-Topology (MLT), which represented by the diagram in Fig. 6 and are defined as

$\mathcal{A}_{\mathrm{MLT}}^{(L)}(p)=\int_{\ell_{1}, \ldots, \ell_{L}} G_{F}\left(q_{1}, \ldots, q_{L+1}\right)$,

where $G_{F}\left(q_{1}, \ldots, q_{L+i}\right)=\prod_{s=1}^{L+1} G_{F}\left(q_{s}\right)$, and $L$ is the number of loops. The momenta of the internal propagators are $q_{s}=\ell_{s}$, with $s \in[1, \ldots, L]$, and $q_{L+1}=-\sum_{s=1}^{L} \ell_{s}+p$. The internal masses, $m_{s}$, are arbitrary. The one-loop twopoint function corresponds to the special case $q_{1}=\ell_{1}$ and $q_{2}=-\ell_{1}+p$. The causal LTD representation of Eq. (61) is extremely compact and is given by

$\mathcal{A}_{\mathrm{MLT}}^{(L)}(p)=-\int_{\ell_{1}, \ldots, \ell_{L}} \frac{1}{x_{L+1}}\left(\frac{1}{\lambda_{L+1}^{-}}+\frac{1}{\lambda_{L+1}^{+}}\right)$, 


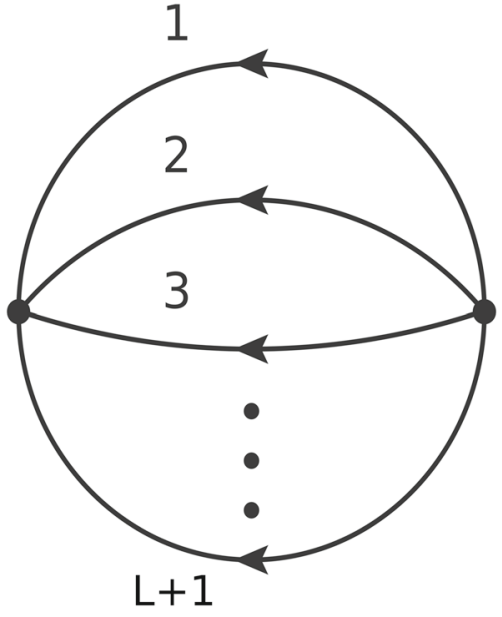

Fig. 6 Maximal Loop Topology at $L$ loops with arbitrary internal masses

where the integration measure in the spacial components of the loop momenta is

$\int_{\ell_{s}}=-\mu^{4-d} \int d^{d-1} \ell_{s} /(2 \pi)^{d-1}$

and

$x_{L+1}=\prod_{s=1}^{L+1} 2 q_{s, 0}^{(+)}, \quad \lambda_{L+1}^{ \pm}=\sum_{s=1}^{L+1} q_{s, 0}^{(+)} \pm p_{0}$,

with $q_{s, 0}^{(+)}=\sqrt{\mathbf{q}_{s}^{2}+m_{s}^{2}-l_{0} 0}$ the on-shell energies and $p_{0}$ the energy component of the external momentum.

The causal representation in Eq. (62) is particularly suitable to achieve the asymptotic expansion in the limit $p^{2} \ll$ $m_{s}^{2}$. Assuming $p=\left(p_{0}, \mathbf{0}\right)$, we obtain

$\mathcal{A}_{\mathrm{MLT}}^{(L)}\left(p^{2} \ll m_{s}^{2}\right)=-2 \sum_{n=0}^{\infty}\left(p^{2}\right)^{n} \int_{\ell_{1}, \ldots, \ell_{L}} \frac{\left(\lambda_{L+1}^{0}\right)^{-1-2 n}}{x_{L+1}}$,

where $\lambda_{L+1}^{0}=\sum_{s=1}^{L+1} q_{s, 0}^{(+)}$. Notice that there is no dependence on $p_{0}$ neither in $x_{L+1}$ nor in $\lambda_{L+1}^{0}$, and therefore the asymptotic integrals on the right-hand side of Eq. (65) are a function of the internal masses exclusively to all loop orders.

\section{Conclusions and outlook}

In this work, we presented several benchmark asymptotic expansions of Feynman integrals in the loop-tree duality formalism. These asymptotic expansions take place at integrand-level in the Euclidean space of the loop threemomentum, where the hierarchies among internal and external scales are more evident than in the Minkowski space of the four-momentum. The method is well-defined since convergence to the full integral is not only achieved in the final result but also at integrand-level, giving ample justification for applying these expansions. Additionally, the UV behaviour of the individual contributions to the asymptotic expansion does not increase when higher orders in the expansion are included. Renormalization is completed locally in four spacetime dimensions with only the first terms of the expansion. Both of these aspects are an improvement compared to the commonly used method of Expansion by Regions.

We have presented explicit results for the scalar two- and three-point functions at one loop in different kinematical limits. Specifically, we have achieved with a single expression a universal description of several asymptotic limits of the twopoint function by conveniently selecting certain parameters of this expression. Recent developments in the realization of the LTD representation at all orders [34] appear especially suitable to facilitating asymptotic expansion. We have provided a final example to all loop orders by exploiting their simplicity.

More work is needed to make a wide range of applications possible at one loop and higher orders leading to additional challenges. Further results and physical applications are underway and will be published in forthcoming publications.

Acknowledgements The project that gave rise to these results received the support of a fellowship from "la Caixa"Foundation (ID 100010434). The fellowship code is LCF/BQ/IN17/11620037. This work is supported by the Spanish Government (Agencia Estatal de Investigación) and ERDF funds from European Commission (Grant No. FPA201784445-P), Generalitat Valenciana (Grant No. PROMETEO/2017/053), Consejo Superior de Investigaciones Científicas (Grant No. PIE201750E021) and the COST Action CA16201 PARTICLEFACE.

Data Availability Statement This manuscript has no associated data or the data will not be deposited. [Authors' comment: This is a theoretical study and does not contain any experimental data.]

Open Access This article is licensed under a Creative Commons Attribution 4.0 International License, which permits use, sharing, adaptation, distribution and reproduction in any medium or format, as long as you give appropriate credit to the original author(s) and the source, provide a link to the Creative Commons licence, and indicate if changes were made. The images or other third party material in this article are included in the article's Creative Commons licence, unless indicated otherwise in a credit line to the material. If material is not included in the article's Creative Commons licence and your intended use is not permitted by statutory regulation or exceeds the permitted use, you will need to obtain permission directly from the copyright holder. To view a copy of this licence, visit http://creativecomm ons.org/licenses/by/4.0/.

Funded by $\mathrm{SCOAP}^{3}$.

\section{References}

1. T. Aoyama et al., The anomalous magnetic moment of the muon in the Standard Model. arXiv:2006.04822 [hep-ph]

2. A. Pich, Flavour anomalies. PoS LHCP2019, 078 (2019). arXiv:1911.06211 [hep-ph] 
3. G. Heinrich, Collider physics at the precision frontier. arXiv:2009.00516 [hep-ph]

4. C.G. Bollini, J.J. Giambiagi, Dimensional renormalization: the number of dimensions as a regularizing parameter. Nuovo Cim. B 12, 20-26 (1972)

5. G. 't Hooft, M.J.G. Veltman, Regularization and renormalization of gauge fields. Nucl. Phys. B 44, 189-213 (1972)

6. X. Chen, T. Gehrmann, E. Glover, M. Jaquier, Precise QCD predictions for the production of Higgs + jet final states. Phys. Lett. B 740, 147-150 (2015). arXiv:1408.5325 [hep-ph]

7. R. Boughezal, F. Caola, K. Melnikov, F. Petriello, M. Schulze, Higgs boson production in association with a jet at next-tonext-to-leading order. Phys. Rev. Lett. 115(8), 082003 (2015). arXiv:1504.07922 [hep-ph]

8. S.P. Jones, M. Kerner, G. Luisoni, Next-to-leading-order QCD corrections to Higgs boson plus jet production with full topquark mass dependence. Phys. Rev. Lett. 120(16), 162001 (2018). arXiv: 1802.00349

9. J.M. Lindert, K. Kudashkin, K. Melnikov, C. Wever, Higgs bosons with large transverse momentum at the LHC. Phys. Lett. B 782, 210 (2018). arXiv:1801.08226

10. M. Beneke, V.A. Smirnov, Asymptotic expansion of Feynman integrals near threshold. Nucl. Phys. B 522, 321 (1998). arXiv:hep-ph/9711391

11. V.A. Smirnov, Applied asymptotic expansions in momenta and masses. Springer Tracts Mod. Phys. 177, 1 (2002)

12. A. Pak, A. Smirnov, Geometric approach to asymptotic expansion of Feynman integrals. Eur. Phys. J. C 71, 1626 (2011). arXiv: 1011.4863

13. B. Jantzen, Foundation and generalization of the expansion by regions. JHEP 12, 076 (2011). arXiv:1111.2589 [hep-ph]

14. B. Jantzen, A.V. Smirnov, V.A. Smirnov, Expansion by regions: revealing potential and Glauber regions automatically. Eur. Phys. J. C 72, 2139 (2012). arXiv: 1206.0546

15. G. Mishima, High-energy expansion of two-loop massive fourpoint diagrams. JHEP 02, 080 (2019). arXiv: 1812.04373 [hep-ph]

16. B. Ananthanarayan, A. Pal, S. Ramanan, R. Sarkar, Unveiling regions in multi-scale Feynman integrals using singularities and power geometry. Eur. Phys. J. C 79(1), 57 (2019). arXiv:1810.06270 [hep-ph]

17. T.Y. Semenova, A.V. Smirnov, V.A. Smirnov, On the status of expansion by regions. Eur. Phys. J. C 79(2), 136 (2019). arXiv: 1809.04325

18. S. Catani, T. Gleisberg, F. Krauss, G. Rodrigo, J.C. Winter, From loops to trees by-passing Feynman's theorem. JHEP 0809, 065 (2008). arXiv:0804.3170

19. I. Bierenbaum, S. Catani, P. Draggiotis, G. Rodrigo, A tree-loop duality relation at two loops and beyond. JHEP 1010, 073 (2010). arXiv:1007.0194

20. I. Bierenbaum, S. Buchta, P. Draggiotis, I. Malamos, G. Rodrigo, Tree-loop duality relation beyond simple poles. JHEP 1303, 025 (2013). arXiv:1211.5048

21. S. Buchta, G. Chachamis, P. Draggiotis, I. Malamos, G. Rodrigo, On the singular behaviour of scattering amplitudes in quantum field theory. JHEP 1411, 014 (2014). arXiv: 1405.7850

22. S. Buchta, Theoretical foundations and applications of the looptree duality in quantum field theories, $\mathrm{PhD}$ thesis, Universitat de València (2015). arXiv:1509.07167

23. S. Buchta, G. Chachamis, P. Draggiotis, G. Rodrigo, Numerical implementation of the loop-tree duality method. Eur. Phys. J. C 77(5), 274 (2017). arXiv: 1510.00187

24. F. Driencourt-Mangin, G. Rodrigo, G.F.R. Sborlini, Universal dual amplitudes and asymptotic expansions for $g g \rightarrow H$ and $H \rightarrow \gamma \gamma$ in four dimensions. Eur. Phys. J. C 78(3), 231 (2018). arXiv:1702.07581 [hep-ph]
25. F. Driencourt-Mangin, G. Rodrigo, G.F.R. Sborlini, W.J. Torres Bobadilla, Universal four-dimensional representation of $H \rightarrow \gamma \gamma$ at two loops through the loop-tree duality. JHEP 1902, 143 (2019). arXiv:1901.09853

26. F. Driencourt-Mangin, Four-dimensional representation of scattering amplitudes and physical observables through the application of the Loop-Tree Duality theorem, $\mathrm{PhD}$ thesis, Universitat de València (2019). arXiv:1907.12450

27. F. Driencourt-Mangin, G. Rodrigo, G.F.R. Sborlini, W.J. Torres Bobadilla, On the interplay between the loop-tree duality and helicity amplitudes. arXiv:1911.11125 [hep-ph]

28. E.T. Tomboulis, Causality and unitarity via the tree-loop duality relation. JHEP 1705, 148 (2017). arXiv:1701.07052

29. R. Runkel, Z. Ször, J.P. Vesga, S. Weinzierl, Causality and loop-tree duality at higher loops. Phys. Rev. Lett. 122(11), 111603 (2019) [Erratum: Phys. Rev. Lett. 123(5), 059902 (2019)]. arXiv: 1902.02135

30. R. Baumeister, D. Mediger, J. Peĉovnik, S. Weinzierl, Vanishing of certain cuts or residues of loop integrals with higher powers of the propagators. Phys. Rev. D 99(9), 096023 (2019). arXiv: 1903.02286 [hep-ph]

31. Z. Capatti, V. Hirschi, D. Kermanschah, B. Ruijl, Loop-tree duality for multiloop numerical integration. Phys. Rev. Lett. 123(15), 151602 (2019). arXiv:1906.06138 [hep-ph]

32. Z. Capatti, V. Hirschi, D. Kermanschah, A. Pelloni, B. Ruijl, Numerical loop-tree duality: contour deformation and subtraction. JHEP 04, 096 (2020). arXiv:1912.09291 [hep-ph]

33. J.J. Aguilera-Verdugo, F. Driencourt-Mangin, J. Plenter, S. Ramírez-Uribe, G. Rodrigo, G.F.R. Sborlini, W .J. Torres Bobadilla, S. Tracz, Causality, unitarity thresholds, anomalous thresholds and infrared singularities from the loop-tree duality at higher orders. JHEP 1912, 163 (2019). arXiv:1904.08389

34. J.J. Aguilera-Verdugo, F. Driencourt-Mangin, R.J. Hernández Pinto, J. Plenter, S.R. Uribe, A.E. Rentería Olivo, G. Rodrigo, G.F. Sborlini, W.J. Torres Bobadilla, S. Tracz, Open loop amplitudes and causality to all orders and powers from the loop-tree duality. Phys. Rev. Lett. 124(21), 211602 (2020). arXiv:2001.03564 [hep-ph]

35. J.J. Aguilera-Verdugo, R.J. Hernandez-Pinto, G. Rodrigo, G.F.R. Sborlini, W.J. Torres Bobadilla, Causal representation of multi-loop amplitudes within the loop-tree duality. arXiv:2006.11217 [hep-ph]

36. S. Ramírez-Uribe, R.J. Hernández-Pinto, G. Rodrigo, G.F.R. Sborlini, W.J. Torres Bobadilla, Universal opening of four-loop scattering amplitudes to trees. arXiv:2006.13818 [hep-ph]

37. C. Gnendiger et al., To $d$, or not to $d$ : recent developments and comparisons of regularization schemes. Eur. Phys. J. C 77(7), 471 (2017). arXiv:1705.01827 [hep-ph]

38. R.J. Hernández-Pinto, G.F.R. Sborlini, G. Rodrigo, Towards gauge theories in four dimensions. JHEP 1602, 044 (2016). arXiv: 1506.04617

39. G.F.R. Sborlini, F. Driencourt-Mangin, R. Hernández-Pinto, G. Rodrigo, Four-dimensional unsubtraction from the loop-tree duality. JHEP 1608, 160 (2016). arXiv: 1604.06699

40. G.F.R. Sborlini, F. Driencourt-Mangin, G. Rodrigo, Fourdimensional unsubtraction with massive particles. JHEP 1610, 162 (2016). arXiv: 1608.01584

41. J. Plenter, Asymptotic expansions through the loop-tree duality. Acta Phys. Polon. B 50, 1983 (2019) 